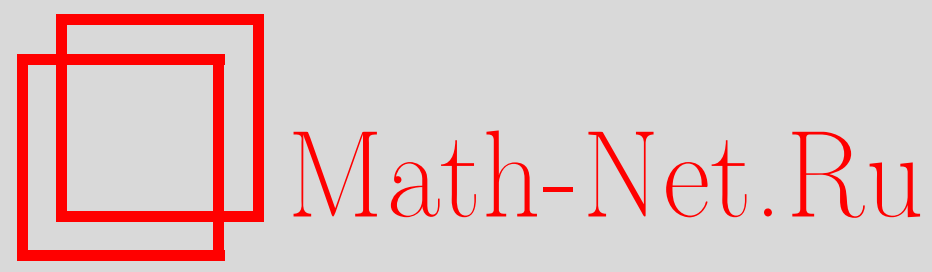

В. Л. Щербина, Об одном случае неразрешимости проблемы эквивалентности программ, Дискрет. матем., 2011, том 23, выпуск 1, 72-83

DOI: https://doi.org/10.4213/dm1131

Использование Общероссийского математического портала Math-Net.Ru подразумевает, что вы прочитали и согласны с пользовательским соглашением http://www . mathnet.ru/rus/agreement

Параметры загрузки:

IP : 3.85 .5 .30

26 апреля 2023 г., $17: 32: 01$ 


\title{
Об одном случае неразрешимости проблемы эквивалентности программ
}

\author{
() 2011 г. В. Л. Щербина
}

\begin{abstract}
В статье рассматриваются алгебраические модели последовательных программ, в которых семантика операторов определяется на основе полугрупп. Впервые построен пример разрешимой полугруппы, обладающей неразложимым нейтральным элементом, для которой проблема останова машины Тьюринга сводится к проблеме эквивалентности программ над указанной полугруппой. Все ранее известные примеры моделей программ с неразрешимой проблемой эквивалентности базировались на группах. Таким образом, в статье удалось уточнить границу между разрешимыми и неразрешимыми случаями проблемы эквивалентности программ в алгебраических моделях. Полученный результат также подтверждает существенность некоторых достаточных условий разрешимости проблемы эквивалентности программ.
\end{abstract}

\section{1. Введение}

Проблема эквивалентности программ является частным случаем задачи проверки программы на соответствие спецификации, если спецификация сама представлена как программа. Например, для проверки корректности оптимизированной версии программы достаточно удостовериться, что поведение неоптимизированной, более простой версии, совпадает (в определенном смысле) с поведением оптимизированного варианта. Рассмотрение поведения программы как частичной функции, ставящей в соответствие входным данным результат вычисления, приводит к проблеме функциональной эквивалентности. Отношение функциональной эквивалентности программ в универсальных вычислительных моделях неразрешимо. Переход к другим моделям вычислений и различные способы определения эквивалентности дают широкий спектр задач, в ряде случаев имеющих разрешающие алгоритмы.

В данной работе рассматривается алгебраическая модель вычислений, изначально предложенная в [5] и развивавшаяся в работах $[6,7,10]$. Формулировка, используемая в данной работе, наиболее близка к $[2,3]$. Модель составлена из трех частей:

- программы (конечной детерминированной системы переходов, явно заданной в виде помеченного графа), которая описывает структуру алгоритма в терминах абстрактных операторов и управляющих ветвлениями логических переменных,

- полугрупповой шкалы, которая определяет всевозможные состояния данных вычислительной системы и то, как выполнение операторов изменяет эти состояния (полугрупповая операция задает воздействие оператора на состояние данных: если 
$s_{1}-$ некоторое состояние данных и $f-$ оператор, то $s_{2}=s_{1} \cdot f-$ состояние данных, которое получится в результате выполнения этого оператора),

- функции оценки, которая определяет зависимость значений логических переменных от текущего состояния данных.

Таким образом, в процессе вычисления программа определяет, какой оператор будет выполняться в зависимости от значений логических переменных в текущем состоянии данных, полугрупповая шкала задает новое состояние данных, полученное в результате выполнения оператора, и функция оценки устанавливает новые значения логических переменных, влияющие на выбор очередного оператора. Результат вычисления - состояние данных, полученное при достижении выделенной заключительной вершины программы.

Две программы считаются эквивалентными относительно фиксированной полугрупповой шкалы, если для любой функции оценки результаты вычисления этих программ совпадают.

В работе [1] доказана теорема, которая гласит, что для шкалы, базирующейся на упорядоченной полугруппе с законом левого сокращения и разрешимой проблемой тождества слов проблема эквивалентности программ разрешима. Построенная в настоящей работе шкала иллюстрирует существенность закона левого сокращения в этой теореме. Место данной работы в ряду других работ, посвященных изучению проблемы эквивалентности программ в сходных моделях, обсуждается в конце следующего раздела, после введения необходимых понятий.

\section{2. Постановка задачи}

Рассмотрим конечный алфавит $\mathscr{A}$, элементы которого назовем операторами. Слова в этом алфавите будут называться операторными цепочками. Для конкатенации слов $w_{1}$ и $w_{2}$ будет использоваться запись $w_{1} w_{2}$, пустое слово будет обозначаться символом $\Lambda$. Запись $|w|$ означает длину слова $w$.

Рассмотрим также непустое конечное множество $\mathscr{C}$, элементами которого являются всевозможные комбинации значений логических переменных (например, это может быть множество всех наборов из нулей и единиц фиксированной длины, отвечающих истинностным значениям предикатов, определенных на состояниях данных).

Программа сигнатуры $(\mathscr{A}, \mathscr{C})$ задается системой переходов $(V$, enter, exit, $B, T)$, где $V$ - конечное множество вершин-преобразователей программы, enter и exit - ocoбые начальная и заключительная вершины (enter, exit $\notin V$ ), $B: V \rightarrow \mathscr{A}$ - функция привязки, сопоставляющая каждому преобразователю программы некоторый оператор, $T:(V \cup\{$ enter $\}) \times C \rightarrow V \cup\{$ exit $\}-$ всюду определенная функция перехода.

Полугрупповой шкалой (или просто шкалой) сигнатуры $\mathscr{A}$ называется тройка $\mathscr{F}=(S, \cdot, \varphi)$, где $(S, \cdot)-$ полугруппа, $\varphi: A^{*} \rightarrow S-$ гомоморфизм свободной полугруппы операторных цепочек с операцией конкатенации на полугруппу $(S, \cdot)$. Будем говорить, что шкала $(S, \cdot, \varphi)$ базируется на полугруппе $(S, \cdot)$. Элементы множества $S$ выполняют две функции: служат состояниями данных и представляют эффект воздействия оператора или серии операторов на состояние данных. Например, состояние данных, полученное при выполнении оператора $а$ в состоянии данных $s$, где $s \in S$ и $a \in \mathscr{A}$, может быть записано как $s \cdot \varphi(a)$. В качестве начального состояния данных возьмем $\varphi(\Lambda)$.

Моделью сигнатуры $(\mathscr{A}, \mathscr{C})$ назовем пару $\mathcal{M}=(\mathscr{F}, \xi)$, в которой $\xi: S \rightarrow \mathscr{C}-$ всюду определенная функция оценки. Понятие вычисления опирается на связь, которую мо- 
дель устанавливает между операторами программы, состояниями данных и значениями логических переменных.

Вычислением программы $\pi=(V$, enter, exit, $B, T)$ сигнатуры $(\mathscr{A}, \mathscr{C})$ в модели $M=(\mathscr{F}, \xi)$ той же сигнатуры называется конечная или бесконечная последовательность пар $r=\left(s_{0}, v_{0}\right),\left(s_{1}, v_{1}\right), \ldots,\left(s_{m}, v_{m}\right), \ldots$, если она удовлетворяет следующим требованиям:

(1) $s_{0}=\varphi(\Lambda), v_{0}=T\left(\right.$ enter, $\left.\xi\left(s_{0}\right)\right)$;

(2) для каждого $i, i \geqslant 1$, выполняются соотношения

$$
s_{i}=s_{i-1} \varphi\left(B\left(v_{i-1}\right)\right), \quad v_{i}=T\left(v_{i-1}, \xi\left(s_{i}\right)\right)
$$

(3) эта последовательность оканчивается парой $\left(s_{m}, v_{m}\right)$ тогда и только тогда, когда $v_{m}=$ exit.

Результатом вычисления назовем элемент $s_{m}$, если вычисление оканчивается парой $\left(s_{m}, v_{m}\right)$, или особое значение $\perp$, если вычисление бесконечно.

Две программы сигнатуры $(\mathscr{A}, \mathscr{C})$ называются эквивалентными на шкале $\mathscr{F}$, если в любой модели $\mathcal{M}=(\mathscr{F}, \xi)$ результаты их вычислений совпадают.

Полугрупповая шкала $(S, \cdot, \varphi)$ называется упорядоченной, если не существует такого состояния данных $s \in S$ и такой непустой операторной цепочки $w \in \mathscr{A}^{+}$, что $s \cdot \varphi(w)=s$.

Полугрупповая шкала $(S, \cdot, \varphi)$ называется уравновешенной, если существует гомоморфизм $t: S \rightarrow \mathbf{N}$ полугрупп $(S, \cdot)$ и $(\mathbf{N},+)$ такой, что $t(\varphi(w))=|w|$ для любого $w \in \mathscr{A}^{*}$. Очевидно, любая уравновешенная шкала является упорядоченной.

Шкалы, базирующиеся на нетривиальных группах, очевидно, являются неупорядоченными. В [4] доказана неразрешимость проблемы эквивалентности программ на шкалах, базирующихся на конечно-порожденных абелевых группах ранга, большего 1, и предлагается разрешающий алгоритм экспоненциальной сложности для проверки эквивалентности на шкале с абелевой группой ранга 1. В [9] показано, что существенно более эффективного алгоритма быть не может: проблема эквивалентности программ на шкале с абелевой группой $(\mathbf{Z},+)$ является PSPACE-полной. В [4] также установлено, что для свободных групп проблема эквивалентности программ является разрешимой (без оценки сложности разрешающего алгоритма).

Насколько известно автору, все полугрупповые шкалы, для которых ранее была доказана неразрешимость проблемы эквивалентности, являются неупорядоченными.

С другой стороны, упорядоченные шкалы обычно приводят к более простым вариантам проблемы эквивалентности программ. Для некоторых упорядоченных (и тем более уравновешенных) шкал проблема эквивалентности может решаться за полиномиальное время. Такие модели исследовались в [2, 3, 8].

К наиболее общим результатам о разрешимости проблемы эквивалентности программ для упорядоченных шкал относится следующая доказанная в [1] теорема.

Теорема 1. Пусть полугрупповая шкала $(S, \cdot, \varphi)$ удовлетворяет следующим требованиям:

(1) в полугруппе $(S, \cdot)$ выполняется закон левого сокращения, то есть для любых элементов $s, s_{1}, s_{2} \in S$, равенство $s \cdot s_{1}=s \cdot s_{2}$ влечет $s_{1}=s_{2}$,

(2) проблема тождества слов разрешима в полугруппе $(S, \cdot)$,

(3) шкала $(S, \cdot, \varphi)$ упорядочена. 
Тогда проблема эквивалентности программ на шкале $(S, \cdot, \varphi)$ разрешима.

Условие 2 существенно, потому что проблема тождества слов сводится в проблеме эквивалентности программ. Существенность условия 3 иллюстрируется с помощью абелевой группы ранга, большего 1 .

В настоящей работе построена шкала, удовлетворяющая условиям 2 и 3 , для которой проблема эквивалентности программ неразрешима. Таким образом, показана существенность условия 1.

\section{3. Машина Тьюринга}

Описываемая полугрупповая шкала устроена таким образом, чтобы в совокупности с подходящей программой моделировать вычисление произвольной машины Тьюринга. В последнем разделе статьи будет показано, каким образом проблема останова машины Тьюринга сводится к проблеме эквивалентности программ на предложенной шкале.

Машину Тьюринга можно описать набором $\left(Q, q_{\text {initial }}, q_{\text {final }}, \delta_{q}, \delta_{a}, \delta_{m}\right)$, где $Q$ - конечное множество состояний, $q_{\text {initial }}$ - начальное состояние, $q_{\text {initial }} \in Q, q_{\text {final }}$ - конечное состояние, $q_{\text {final }} \notin Q$, и $\delta_{q}: Q \times\{0,1\} \rightarrow Q \cup\left\{q_{\text {final }}\right\}-$ функция перехода в новое состояние, $\delta_{a}: Q \times\{0,1\} \rightarrow\{0,1\}-$ функция записи на ленту, $\delta_{m}: Q \times\{0,1\} \rightarrow\{-1,0,1\}-$ функция сдвига, причем эти функции всюду определены.

Вычислением машины Тьюринга (в работе рассматриваются только вычисления на изначально пустой ленте) назовем конечную или бесконечную последовательность четверок $\left(q_{0}, f_{0}, b_{01}, b_{02}\right),\left(q_{1}, f_{1}, b_{11}, b_{12}\right), \ldots,\left(q_{n}, f_{n}, b_{n 1}, b_{n 2}\right), \ldots$, где $f_{i}: \mathbf{Z} \rightarrow\{0,1\}$, $q_{i} \in Q \cup\left\{q_{\text {final }}\right\}, b_{i 1}, b_{i 2} \in \mathbf{N}$, удовлетворяющую следующим требованиям:

$-f_{0}(\cdot)=0, b_{01}=b_{02}=0, q_{0}=q_{\text {initial }}$,

- каждый член последовательности, кроме первого, связан с предыдущим соотношениями

$$
\begin{aligned}
q_{i+1} & =\delta_{q}\left(q_{i}, f_{i}(0)\right), \\
f_{i+1}\left(x-\delta_{m}\left(q_{i}, f_{i}(0)\right)\right) & = \begin{cases}\delta_{a}\left(q_{i}, f_{i}(0)\right), & x=0, \\
f_{i}(x), & x \neq 0,\end{cases} \\
b_{i+1,1} & =\max \left(b_{i 1}+\delta_{m}\left(q_{i}, f_{i}(0)\right), 0\right), \\
b_{i+1,2} & =\max \left(b_{i 2}-\delta_{m}\left(q_{i}, f_{i}(0)\right), 0\right),
\end{aligned}
$$

- последовательность заканчивается элементом $\left(q_{n}, f_{n}, b_{n 1}, b_{n 2}\right)$ тогда и только тогда, когда $q_{n}=q_{\text {final }}$.

Значение $q_{i}$ в этом определении - текущее состояние машины, значение $f_{i}(x)$ есть символ, записанный в ячейку с номером $x$, отсчитывая от обозреваемой ячейки, а $b_{i 1}$ и $b_{i 2}$ - протяженности рабочей зоны ленты влево и вправо от обозреваемой ячейки соответственно.

\section{4. Структура полугруппы}

В сигнатуре $(\mathscr{A}, \mathscr{C})$ описываемой шкалы множество операторов имеет вид

$$
\mathscr{A}=\left\{\$, a_{0}, a_{1}, \hat{a}_{0}, \hat{a}_{1}, l, r, d, a_{0}^{!}, a_{1}^{!}, a_{0}^{?}, a_{1}^{?}, E\right\},
$$


а множество комбинаций значений логических переменных состоит из двух элементов: $\mathscr{C}=\{0,1\}$ (можно считать, что в программе допустима только одна логическая переменная).

Опишем полугрупповую шкалу $\mathscr{F}=(S, \cdot, \varphi)$.

Полугруппа $(S, \cdot)$ задана своим конечным представлением: ее порождающие элементы - операторы из множества $\mathcal{A}$, а определяющие соотношения (для всевозможных $\left.x \in\left\{\$, a_{0}, a_{1}\right\}, y \in\left\{l, r, d, a_{0}^{!}, a_{1}^{!}, a_{0}^{?}, a_{1}^{?}\right\}, z \in \mathcal{A}, i, j \in\{0,1\}\right)$ таковы:

$$
\begin{aligned}
a_{j} \cdot \hat{a}_{i} \cdot l & =\hat{a}_{j} \cdot a_{i} \cdot d, \\
\$ \cdot \hat{a}_{i} \cdot l & =\$ \cdot \hat{a}_{0} \cdot a_{i}, \\
\hat{a}_{i} \cdot a_{j} \cdot r & =a_{i} \cdot \hat{a}_{j} \cdot d, \\
\hat{a}_{i} \cdot \$ \cdot r & =a_{i} \cdot \hat{a}_{0} \cdot \$, \\
\hat{a}_{i} \cdot a_{j}^{!} & =\hat{a}_{j} \cdot d, \\
\hat{a}_{i} \cdot a_{i}^{?} & =\hat{a}_{i} \cdot d, \\
\hat{a}_{i} \cdot a_{1-i}^{?} & =E \cdot E, \\
E \cdot z=z \cdot E & =E \cdot E, \\
x \cdot y & =y \cdot x, \\
d \cdot y & =y \cdot d .
\end{aligned}
$$

Отображение $\varphi: \mathscr{A}^{*} \rightarrow S$ однозначно определяется следующими требованиями:

- на множестве $\mathscr{A}$ оно должно совпадать с тождественным отображением,

- для любых операторных цепочек $w_{1}, w_{2} \in A^{*}$ выполнено соотношение

$$
\varphi\left(w_{1} w_{2}\right)=\varphi\left(w_{1}\right) \cdot \varphi\left(w_{2}\right)
$$

Символы $\$, a_{0}, a_{1}, \hat{a}_{0}, \hat{a}_{1}$ предназначены для кодирования содержимого ленты и положения обозреваемой ячейки: $\$-$ граница рабочей зоны ленты, $\hat{a}_{i}-$ обозреваемая ячейка, содержащая символ $i$, где $i \in\{0,1\}, a_{i}$ - любая другая ячейка рабочей зоны, содержащая символ $i$. Таким образом, содержимое ленты может кодироваться операторными цепочками с помощью функции tape: $(\mathbf{Z} \rightarrow\{0,1\}) \times \mathbf{N} \times \mathbf{N} \rightarrow \mathscr{A}^{*}$, заданной соотношением

$$
\operatorname{tape}\left(f, b_{1}, b_{2}\right)=\$ a_{f\left(-b_{1}\right)} a_{f\left(-b_{1}+1\right)} \ldots a_{f(-2)} a_{f(-1)} \hat{a}_{f(0)} a_{f(1)} a_{f(2)} \ldots a_{f\left(b_{2}-1\right)} a_{f\left(b_{2}\right)} \$ .
$$

Область значений этой функции обозначим Tapes.

Операторы из множества Commands $=\left\{l, r, a_{0}^{!}, a_{1}^{!}, d\right\}$ предназначены для моделирования операций, которые может совершить машина Тьюринга: перемещение головки влево и вправо, запись 0 или 1 в обозреваемую ячейку, пустое действие (задержка). Операторы $a_{i}^{?}$, где $i \in\{0,1\}$, моделируют сравнение содержимого обозреваемой ячейки со значением $i$. Если в обозреваемой ячейке записано значение $i$, то выполнение оператора $a_{i}^{?}$ изменяет состояние данных таким же образом, как и выполнение оператора задержки $d$. В противном случае, выполнение оператора $a_{i}^{?}$ приводит к состоянию из класса Err $=\left\{\varphi\left(E^{n+1}\right) \mid n \in \mathbf{N}\right\}$.

Такое поведение операторов выражено в определяющих соотношениях. Тождество (1) определяет семантику оператора $l$ (перемещение головки влево на одну ячейку). Элемент $d$ (задержка) вводится для сохранения длины представления (для обеспечения свойства 
уравновешенности шкалы). Соотношение (2) соответствует ситуации, когда головка достигла левой границы рабочей зоны ленты, и рабочая зона должна быть расширена. Аналогично соотношения (3) и (4) описывают оператор $r$. Тождество (5) определяет операторы записи на ленту, тождество (6) характеризует успешное сопоставление содержимого обозреваемой ячейки (когда в ячейке записано ожидаемое значение), тождество (7) неуспешное (за счет оператора $E$ состояние данных может быть отнесено к классу Err в силу тождества (8)).

Соотношения (9) и (10) в совокупности с ранее описанными соотношениями отождествляют состояния данных вида $\varphi\left(t d^{n}\right) \cdot \varphi(c)$ и $\varphi\left(t^{\prime} d^{m}\right)$, где $t-$ закодированное содержимое ленты до выполнения операции $c \in$ Commands, $t^{\prime}-$ закодированное состояние ленты после выполнения этой операции, $n \in \mathbf{N}$ и $m=n+|t|+1-\left|t^{\prime}\right|$.

Лемма 1. Никакой элемент полугруппь $(S, \cdot)$ вида $\varphi\left(t d^{m}\right)$, где $t \in$ Tapes, $m \in \mathbf{N}$, не принадлежит классу Err.

Доказательство. Далее условимся обозначать символом $w$ (возможно, с индексами) произвольные операторные цепочки, символом $c$ - элементы множества Commands $\backslash\{d\}$, символом $\bar{c}$ - операторные цепочки, состоящие только из элементов множества Commands $\backslash\{d\}$, символом $t-$ цепочки из множества Tapes, символами $m$ и $n-$ натуральные числа.

Будем рассматривать определяющие соотношения как правила преобразования операторных цепочек. Нужно показать, что с помощью этих правил из цепочки $t d^{m}$ невозможно получить цепочку, содержащую оператор $E$.

Определим отношение эквивалентности ( ) на множестве операторных цепочек: две цепочки считаются эквивалентными тогда и только тогда, когда одну из них можно получить из другой с помощью серии преобразований, заданных структурными тождествами (9) и (10). Выражение $[w]$ обозначает класс эквивалентности, содержащий цепочку $w$.

Особый интерес для доказательства представляют цепочки из множества

$$
W=\left\{w: w \sim t \bar{c} d^{m}\right\} .
$$

Легко проверить, что из того, что $t_{1} \bar{c}_{1} d^{m_{1}} \neq t_{2} \bar{c}_{2} d^{m_{2}}$ следует, что $\left[t_{1} \bar{c}_{1} d^{m_{1}}\right] \neq\left[t_{2} \bar{c}_{2} d^{m_{2}}\right]$.

Определим частичную функцию content: $\mathscr{A}^{*} \rightarrow(\mathbf{Z} \rightarrow\{0,1\})$, полагая

$$
\operatorname{content}\left(\$ a_{i_{-b_{1}}} a_{i_{-b_{1}+1}} \ldots a_{i_{-2}} a_{i_{-1}} \hat{a}_{i_{0}} a_{i_{1}} a_{i_{2}} \ldots a_{i_{b_{2}-1}} a_{i_{b_{2}}} \$\right)=f
$$

где

$$
f(k)= \begin{cases}i_{k}, & -b_{1} \leqslant k \leqslant b_{2}, \\ 0 & \text { в противном случае. }\end{cases}
$$

Определим функцию act: $(\mathbf{Z} \rightarrow\{0,1\}) \times($ Commands $\backslash\{d\}) \rightarrow(\mathbf{Z} \rightarrow\{0,1\})$, полагая

$$
\operatorname{act}(f, c)=f_{c},
$$

где

$$
\begin{aligned}
f_{a_{i}^{!}}(k) & = \begin{cases}i, & k=1, \\
f(k), & k \neq 1,\end{cases} \\
f_{a_{i}^{?}} & =f, \\
f_{l}(k) & =f(k-1), \\
f_{r}(k) & =f(k+1) .
\end{aligned}
$$


Ее можно естественным образом расширить до функции

$$
\text { act }^{*}:(\mathbf{Z} \rightarrow\{0,1\}) \times(\text { Commands } \backslash\{d\})^{*} \rightarrow(\mathbf{Z} \rightarrow\{0,1\})
$$

полагая

$$
\begin{aligned}
\operatorname{act}^{*}(f, \Lambda) & =f, \\
\operatorname{act}^{*}(f, c \bar{c}) & =\operatorname{act}^{*}(\operatorname{act}(f, c), \bar{c}) .
\end{aligned}
$$

Определим на множестве $\mathscr{A}$ бинарные отношения $\left.(\stackrel{l}{\rightarrow}),(\stackrel{r}{\rightarrow}),\left(\stackrel{a_{0}^{!}}{\rightarrow}\right), \stackrel{a_{1}^{!}}{\rightarrow}\right),\left(\stackrel{a_{0}^{?}}{\rightarrow}\right),\left(\stackrel{a_{1}^{?}}{\rightarrow}\right)$. Отношение $w_{1} \stackrel{l}{\rightarrow} w_{2}$ имеет место тогда и только тогда, когда цепочка $w_{2}$ может быть получена из цепочки $w_{1}$ заменой подцепочки $a_{j} \hat{a}_{i} l$ на подцепочку $\hat{a}_{j} a_{i} d$ (то есть левой части тождества (1) на правую), либо $\$ \hat{a}_{i} l$ на $\$ \hat{a}_{0} a_{i}$ (то есть левой части тождества (2) на правую). Аналогично задается отношение $(\stackrel{r}{\rightarrow})$. Запись $w_{1} \stackrel{a_{j}^{!}}{\rightarrow} w_{2}$ означает, что $w_{2}$ получается из $w_{1}$ однократной заменой $\hat{a}_{i} a_{j}^{!}$на $\hat{a}_{j} d$, а $w_{1} \stackrel{a_{i}^{?}}{\rightarrow} w_{2}$ тогда и только тогда, когда $w_{2}$ получается из $w_{1}$ заменой $\hat{a}_{i} a_{i}^{?}$ на $\hat{a}_{i} d$. Введем также обозначения $(\stackrel{l}{\leftarrow})=(\stackrel{l}{\rightarrow})^{-1}$, $(\stackrel{r}{\leftarrow})=(\stackrel{r}{\rightarrow})^{-1}$ и т. д. Отношение $(\stackrel{l}{\rightarrow}) \cup(\stackrel{r}{\rightarrow}) \cup\left(\stackrel{a_{0}^{!}}{\rightarrow}\right) \cup\left(\stackrel{a_{1}^{!}}{\rightarrow}\right) \cup\left(\stackrel{a_{0}^{?}}{\rightarrow}\right) \cup\left(\stackrel{a_{1}^{?}}{\rightarrow}\right)$ обозначим символом $(\rightarrow)$, а отношение $(\rightarrow) \cup(\rightarrow)^{-1}-$ символом $(\leftrightarrow)$.

Так как для любых операторных цепочек $w_{1}, w_{2}, w_{3}, w_{4} \in W$ соотношения $w_{1} \sim w_{3}$, $w_{1} \stackrel{l}{\rightarrow} w_{2}, w_{3} \stackrel{l}{\rightarrow} w_{4}$ влекут $w_{2} \sim w_{4}$, естественно распространить отношение $(\stackrel{l}{\rightarrow})$ (и аналогично другие отношения) на классы эквивалентности:

$$
\left[w_{1}\right] \stackrel{l}{\rightarrow}\left[w_{2}\right] \Longleftrightarrow \exists w_{3} \in\left[w_{1}\right] \quad \exists w_{4} \in\left[w_{2}\right] \quad w_{3} \stackrel{l}{\rightarrow} w_{4}
$$

Из соотношения $\left[t_{1} \bar{c}_{1} d^{m_{1}}\right] \stackrel{c}{\rightarrow}\left[t_{2} \bar{c}_{2} d^{m_{2}}\right]$ следуют равенства

$$
\begin{aligned}
\bar{c}_{1} & =c \bar{c}_{2}, \\
\operatorname{content}\left(t_{2}\right) & =\operatorname{act}\left(\operatorname{content}\left(t_{1}\right), c\right) .
\end{aligned}
$$

Докажем это утверждение для случая $c=l$. Из структуры определяющих тождеств следует, что цепочки $w_{1} \in\left[t_{1} \bar{c}_{1} d^{m_{1}}\right]$ и $w_{2} \in W$ такие, что $w_{1} \stackrel{c}{\rightarrow} w_{2}$, имеют либо вид

$$
\begin{aligned}
& w_{1}=\$ a_{i_{-b_{1}}} a_{i_{-b_{1}+1}} \ldots a_{i_{-2}} a_{i_{-1}} \hat{a}_{i_{0}} l w, \\
& w_{2}=\$ a_{i_{-b_{1}}} a_{i_{-b_{1}+1}} \ldots a_{i_{-2}} a_{i_{-1}} a_{i_{0}} d w,
\end{aligned}
$$

либо вид

$$
\begin{aligned}
& w_{1}=\$ \hat{a}_{i_{0}} l w, \\
& w_{2}=\$ \hat{a_{0}} a_{i_{0}} w,
\end{aligned}
$$

где

$$
w \sim a_{i_{1}} a_{i_{2}} \ldots a_{i_{b_{2}-1}} a_{i_{b_{2}}} \$ d^{m_{1}}
$$


И в том, и в другом случае легко удостовериться, что

$$
w_{2} \sim t_{3} \bar{c}_{3} d^{m_{3}}
$$

для некоторой цепочки $t_{3} \bar{c}_{3} d^{m_{3}}$ такой, что

$$
\begin{aligned}
\bar{c}_{1} & =c \bar{c}_{3}, \\
\operatorname{content}\left(t_{3}\right) & =\operatorname{act}\left(\operatorname{content}\left(t_{1}\right), c\right) .
\end{aligned}
$$

Как уже было замечено, соотношение $t_{3} \bar{c}_{3} d^{m_{3}} \sim t_{2} \bar{c}_{2} d^{m_{2}}$ влечет $t_{3} \bar{c}_{3} d^{m_{3}}=t_{2} \bar{c}_{2} d^{m_{2}}$, а значит, для цепочки $t_{2} \bar{c}_{2} d^{m_{2}}$ также справедливы равенства

$$
\begin{aligned}
\bar{c}_{1} & =c \bar{c}_{2}, \\
\operatorname{content}\left(t_{2}\right) & =\operatorname{act}\left(\operatorname{content}\left(t_{1}\right), c\right) .
\end{aligned}
$$

Случаи других значений $c$ исследуются аналогично.

Под серией преобразований будем понимать последовательность $\left[w_{1}\right],\left[w_{2}\right], \ldots,\left[w_{n}\right]$, где $w_{i}=t_{i} \bar{c}_{i} d^{m_{i}}$, в которой $\left[w_{i}\right] \leftrightarrow\left[w_{i+1}\right]$ для всех $i$ таких, что $1 \leqslant i<n$. Для любой серии преобразований имеет место следующее утверждение: если выполняются соотношения $\bar{c}_{1}=\bar{c}_{n} u\left|\bar{c}_{i}\right| \geqslant\left|\bar{c}_{1}\right|$ для всех $i$ таких, что $1<i<n$, mо content $\left(t_{1}\right)=\operatorname{content}\left(t_{n}\right)$. Докажем это утверждение с помощью индукции по длине серии преобразований. Очевидно, длина такой последовательности нечетна. Для одноэлементной последовательности утверждение, очевидно, истинно. Если $n \geqslant 3$, возможны два случая.

(1) Для всех $i$ таких, что $2 \leqslant i \leqslant n-1$, выполняется неравенство $\left|\bar{c}_{i}\right|>\left|\bar{c}_{1}\right|$. Легко проверить, что $\left[w_{1}\right] \stackrel{c}{\leftarrow}\left[w_{2}\right]$ и $\left[w_{n-1}\right] \stackrel{c}{\rightarrow}\left[w_{n}\right]$, а значит, $\bar{c}_{2}=\bar{c}_{n-1}=c \bar{c}_{1}$ для некоторого $c \in($ Commands $\backslash\{d\})$. Воспользуемся предположением индукции для серии преобразований $\left[w_{2}\right], \ldots,\left[w_{n-1}\right]$ и равенствами $\operatorname{content}\left(t_{1}\right)=\operatorname{act}\left(\operatorname{content}\left(t_{2}\right), c\right)$, $\operatorname{content}\left(t_{n}\right)=\operatorname{act}\left(\operatorname{content}\left(t_{n-1}\right), c\right)$.

(2) $\left|\bar{c}_{j_{1}}\right|=\left|\bar{c}_{j_{2}}\right|=\ldots=\left|\bar{c}_{j_{m}}\right|=\left|\bar{c}_{1}\right|$, где индексы $j_{1}, j_{2}, \ldots, j_{m}$ таковы, что $1=j_{1}<$ $j_{2}<\ldots<j_{m}=n$, и для всех $i \in[1, n] \backslash\left\{j_{1}, \ldots, j_{m}\right\}$ выполняется неравенство $\left|\bar{c}_{i}\right|>\left|\bar{c}_{1}\right|$. Отсюда следуют равенства $\bar{c}_{j_{1}}=\bar{c}_{j_{2}}=\ldots=\bar{c}_{j_{m}}=\bar{c}_{1}$, поэтому, пользуясь индуктивной гипотезой для серий $\left[w_{i_{k}}\right],\left[w_{i_{k}+1}\right], \ldots,\left[w_{i_{k+1}}\right]$ при $k \in[1, n]$, устанавливаем цепочку равенств content $\left(t_{i_{1}}\right)=\operatorname{content}\left(t_{i_{2}}\right)=\ldots=\operatorname{content}\left(t_{i_{n}}\right)$.

Для завершения доказательства леммы нужно обосновать следующее утверждение. Допустим, что $\left[w_{1}\right],\left[w_{2}\right], \ldots,\left[w_{n}\right]-$ серия преобразований, где $w_{i}=t_{i} \bar{c}_{i} d^{m_{i}}$, причем $\bar{c}_{1}=\Lambda$ и $\bar{c}_{n}$ начинается с $a_{j}$. Тогда content $\left(t_{n}\right)(0)=j$.

Действительно, рассмотрим наибольшее $i$ такое, что $\left|\bar{c}_{i-1}\right|=\left|\bar{c}_{n}\right|-1$ и $\left|\bar{c}_{i}\right|=\left|\bar{c}_{n}\right|$. Очевидно, что $\left[w_{i-1}\right] \stackrel{a_{j}^{?}}{\leftarrow}\left[w_{i}\right]$, а значит, $\operatorname{content}\left(t_{i}\right)(0)=j$. Но для всех $k$ из $[i+1, n-1]$ справедливо неравенство $\left|\bar{c}_{k}\right|>\left|\bar{c}_{i}\right|$, а значит, content $\left(t_{i}\right)=\operatorname{content}\left(t_{n}\right)$.

\section{5. Моделирование машины Тьюринга}

Пусть $M=\left(Q, q_{\text {initial }}, q_{\text {final }}, \delta_{q}, \delta_{a}, \delta_{m}\right)$ - произвольная машина Тьюринга. Построим моделирующую ее программу $\pi_{M}=(V$, enter, exit, $B, T)$ сигнатуры $(\mathscr{A}, \mathscr{C})$.

В качестве множества вершин-преобразователей возьмем множество

$$
V=Q \times\{0,1\} \times\{0,1,2\} \cup\left\{v_{1}, v_{2}, v_{3}, v_{4}\right\},
$$


где $v_{1}, v_{2}, v_{3}$ и $v_{4}-$ четыре произвольных попарно различных элемента, не принадлежащих множеству $Q \times\{0,1\} \times\{0,1,2\}$. Функция $B$ будет задана соотношениями

$$
\begin{aligned}
& B\left(v_{1}\right)=B\left(v_{3}\right)=\$, \\
& B\left(v_{2}\right)=\hat{a}_{0}, \\
& B((q, i, 0))=a_{i}^{\text {? }}, \\
& B((q, i, 1))=a_{\delta_{a}(q, i)}^{!}, \\
& B((q, i, 2))= \begin{cases}l, & \delta_{m}(q, i)=-1, \\
d, & \delta_{m}(q, i)=0, \\
r, & \delta_{m}(q, i)=1,\end{cases} \\
& B\left(v_{4}\right)=d \text {. }
\end{aligned}
$$

Функция переходов описывается равенствами

$$
\begin{aligned}
T(\text { enter }, \cdot) & =v_{1}, \\
T\left(v_{1}, \cdot\right) & =v_{2}, \\
T\left(v_{2}, \cdot\right) & =v_{3}, \\
T\left(v_{3}, i\right) & =\left(q_{\text {initial }}, i, 0\right), \\
T((q, i, 0), \cdot) & =(q, i, 1), \\
T((q, i, 1), \cdot) & =(q, i, 2), \\
T((q, i, 2), j) & = \begin{cases}\left(\delta_{q}(q, i), j, 0\right), & \delta_{q}(q, i) \neq q_{\text {final }}, \\
v_{4}, & \delta_{q}(q, i)=q_{\text {final }},\end{cases} \\
T\left(v_{4}, \cdot\right) & =\text { exit. }
\end{aligned}
$$

Содержательно каждому переходу машины Тьюринга ставится в соответствие цепочка из трех вершин программы. Специальные вершины $v_{1}, v_{2}$ и $v_{3}$ добавляются в начало программы, вершина $v_{4}-$ в конец. На рис. 1 изображены фрагменты программы $\pi_{M}$. Фрагмент (a) предназначен для получения состояния данных, моделирующего пустую ленту. Фрагмент (b) отвечает переходу машины Тьюринга из состояния $q$ при чтении символа $i$ на ленте. Такие фрагменты присутствуют в программе парами: для символа 0 и для символа 1. На выбор одного из этих двух фрагментов при вычислении влияет только значение функции оценки в текущем состоянии данных (кодирующем содержимое

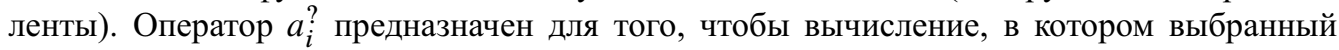
фрагмент не соответствует символу на ленте, приводило к состоянию из множества Err. Далее вводится понятие естественной функции оценки, заведомо приводящей к выбору нужного фрагмента. Оператор $a_{\delta_{a}(q, i)}$ отвечает записи нового символа в текущую ячейку, а оператор $r, l$, или $d-$ перемещению головки машины Тьюринга. Фрагмент (c) является заключительным. Он содержит вершину $v_{4}$, которой приписан оператор $d$ (не влияющий на закодированное в состоянии данных содержимое ленты) в программе $\pi_{M}$ и оператор $E$ в описанной далее модификации этой программы $\pi_{M}^{\prime}$. Программа $\pi_{M}$ устроена таким образом, что либо тройки шагов ее вычисления соответствуют шагам вычисления машины Тьюринга $M$, либо вычисление завершается с результатом из множества Err (или не завершается вовсе). Это свойство отражено в следующих определениях и леммах. 


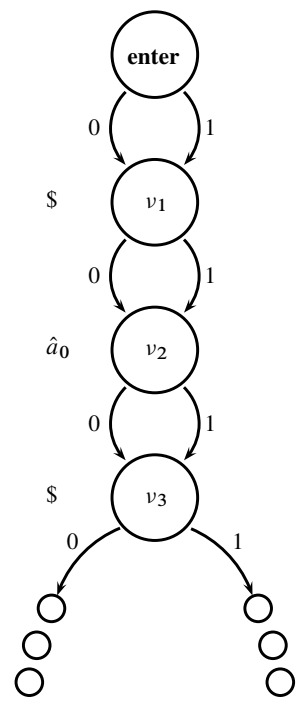

(a)

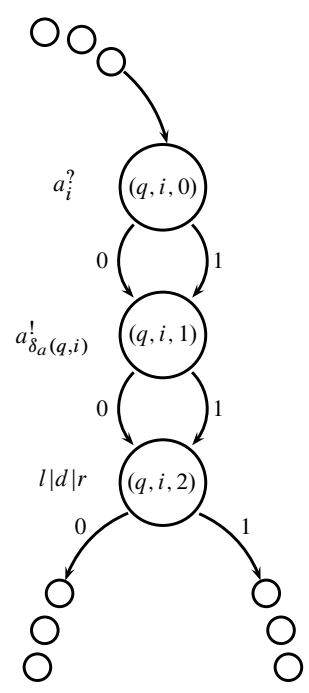

(b)

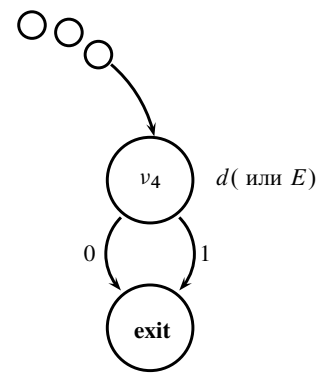

(c)

Рис. 1.

Назовем естественной функцию оценки $\xi: S \rightarrow\{0,1\}$, удовлетворяющую требованию

$$
\xi\left(\varphi\left(\operatorname{tape}\left(f, b_{1}, b_{2}\right) d^{n}\right)\right)=f(0)
$$

для всевозможных $f: \mathbf{Z} \rightarrow\{0,1\}$ и $b_{1}, b_{2}, n \in \mathbf{N}$. Напомним, что множество $S$ - это носитель полугруппы, на которой базируется построенная в предыдущем разделе шкала $\mathscr{F}$.

Покажем, что естественные функции оценки существуют, то есть требования, налагаемые на функцию $\xi$, непротиворечивы. Действительно, пусть справедливо равенство

$$
\varphi\left(\operatorname{tape}\left(f_{1}, b_{11}, b_{12}\right) d^{n_{1}}\right)=\varphi\left(\operatorname{tape}\left(f_{2}, b_{21}, b_{22}\right) d^{n_{2}}\right)=s,
$$

и при этом $f_{1}(0) \neq f_{2}(0)$. Рассмотрим элемент $s^{\prime}=s \cdot a_{f_{1}(0)}^{\text {? }}$. С одной стороны, он равен $\varphi\left(\operatorname{tape}\left(f_{1}, b_{11}, b_{12}\right) d^{n_{1}} a_{f_{1}(0)}^{\text {? }}\right)$, то есть из тождеств (10), (9) и (6) следует, что

$$
s^{\prime}=\varphi\left(\operatorname{tape}\left(f_{1}, b_{11}, b_{12}\right) d^{n_{1}+1}\right),
$$

а значит, $s^{\prime} \notin$ Err в силу леммы 1. С другой стороны,

$$
s^{\prime}=\varphi\left(\operatorname{tape}\left(f_{2}, b_{21}, b_{22}\right) d^{n_{2}} a_{1-f_{2}(0)}^{?}\right),
$$

откуда, в силу тождеств (10), (9), (7) и (8) следует, что

$$
s^{\prime}=\varphi\left(E^{b_{21}+b_{22}+n_{2}+4}\right) \text {. }
$$

Получаем противоречие.

Естественной моделью назовем модель $\mathcal{M}=(\mathscr{F}, \xi)$, где $\xi-$ естественная функция оценки. 
Лемма 2. Если вычисление машины Тьюринга $M$ конечно, то вычисление программы $\pi_{M}$ в естественной модели $\mathcal{M}$ конечно и результат его не принадлежит множеству Err. Если вычисление этой машины Тьюринга бесконечно, то и вычисление программы $\pi_{M} 8$ модели М бесконечно.

Доказательство. Легко проверяется, что если вычисление машины Тьюринга $M$ имеет вид $\left(q_{0}, f_{0}, b_{01}, b_{02}\right),\left(q_{1}, f_{1}, b_{11}, b_{12}\right), \ldots,\left(q_{i}, f_{i}, b_{i, 1}, b_{i, 2}\right), \ldots,\left(q_{n}, f_{n}, b_{n, 1}, b_{n, 2}\right), \ldots$, то вычисление программы $\pi_{M}$ в модели $\mathcal{M}$ может быть записано в виде $\left(\varphi(\Lambda), v_{1}\right),\left(\varphi(\$), v_{2}\right)$, $\left(\varphi\left(\$ \hat{a}_{0}\right), v_{3}\right),\left(\varphi\left(\operatorname{tape}\left(f_{0}, b_{01}, b_{02}\right)\right),\left(q_{0}, f_{0}(0), 0\right)\right),\left(\varphi\left(\operatorname{tape}\left(f_{0}, b_{01}, b_{02}\right) a_{f_{0}(0)}^{?}\right),\left(q_{0}, f_{0}(0), 1\right)\right)$, $\left(\varphi\left(\operatorname{tape}\left(f_{0}, b_{01}, b_{02}\right) a_{f_{0}(0)}^{?} a_{\delta_{a}\left(q_{0}, f_{0}(0)\right)}^{!}\right),\left(q_{0}, f_{0}(0), 2\right)\right),\left(\varphi\left(\operatorname{tape}\left(f_{1}, b_{11}, b_{12}\right) d^{m_{10}}\right),\left(q_{1}, f_{1}(0)\right.\right.$, $0))), \quad \ldots, \quad\left(\varphi\left(\operatorname{tape}\left(f_{i}, b_{i, 1}, b_{i, 2}\right) d^{m_{i 0}}\right),\left(q_{i}, f_{i}(0), 0\right)\right), \quad\left(\varphi\left(\operatorname{tape}\left(f_{i}, b_{i, 1}, b_{i, 2}\right) a_{f_{i}(0)} d^{m_{i 1}}\right),\left(q_{i}\right.\right.$,

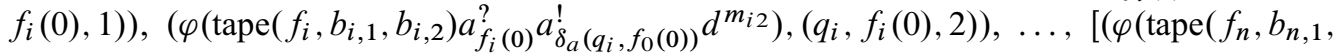
$\left.\left.\left.b_{n, 2}\right) d^{m_{n 0}}\right), v_{4}\right),\left(\varphi\left(\operatorname{tape}\left(f_{n}, b_{n, 1}, b_{n, 2}\right) d^{m_{n 1}}\right)\right.$, exit)]. В этой записи заключенная в квадратные скобки часть присутствует только в том случае, когда вычисление конечно. Из леммы 1 непосредственно следует, что $\varphi\left(\operatorname{tape}\left(f_{n}, b_{n, 1}, b_{n, 2}\right) d^{m_{n 1}}\right) \notin$ Err.

Из доказательства видно, что вычисления программы в различных естественных моделях совпадают.

Лемма 3. Если конечное вычисление программы $\pi_{M}$, построенной по машине Тьюринга $M$, в модели $M=(\mathscr{F}, \xi)$, где $\xi$ - произвольная функция оценки, отличается от вычисления программы в естественной модели, то его результат принадлежит множеству Err.

Доказательство. Переходы $T(v, 0)$ и $T(v, 1)$ ведут в различные вершины только из вершин $v$, принадлежащих множеству $\left\{v_{3}\right\} \cup\left\{(q, i, 2) \mid q \in Q, \delta_{q}(q, i) \neq q_{\text {final }}\right\}$. Значит, вычисление программы $\pi_{M}$ в модели $\mathcal{M}=(\mathscr{F}, \xi)$ имеет вид $\left(s_{0}, v_{0}\right),\left(s_{1}, v_{1}\right), \ldots,\left(s_{m}, v_{m}\right)$, $\left(s_{m+1},(q, j, 0)\right),\left(s_{m+1} \cdot a_{j}^{?},(q, j, 1)\right), \ldots$, а вычисление этой программы в естественной модели имеет вид $\left(s_{0}, v_{0}\right),\left(s_{1}, v_{1}\right), \ldots,\left(s_{m}, v_{m}\right),\left(s_{m+1},(q, 1-j, 0)\right),\left(s_{m+1} \cdot a_{1-j}^{\text {? }}\right.$, $(q, 1-j, 1)), \ldots$, где $v_{m} \in\left\{v_{3}\right\} \cup\left\{(q, i, 2) \mid q \in Q, \delta_{q}(q, i) \neq q_{\text {final }}\right\}$.

Для завершения доказательства заметим, что соотношение $\varphi\left(t d^{n} a_{j}^{?}\right) \notin$ Err, где $t \in$ Tapes, влечет справедливость включения $\varphi\left(t d^{n} a_{1-j}^{?}\right) \in$ Err.

Лемма 4. Модель вида $\mathcal{M}=(\mathscr{F}, \xi)$, в которой программа $\pi_{M}$ имеет конечное вычисление с результатом, не принадлежащим множеству Err, существует тогда и только тогда, когда вычисление машины Тьюринга $M$ завершается.

Доказательство. Если вычисление машины Тьюринга $M$ завершается, то в качестве модели $\mathcal{M}$ можно взять любую естественную модель. Если вычисление машины Тьюринга $M$ не завершается, то вычисление программы $\pi_{M}$ в естественной модели бесконечно (по лемме 2), а любое другое вычисление этой программы завершается с результатом из множества Err (по лемме 3). Лемма доказана.

Рассмотрим модификацию $\pi_{M}^{\prime}=\left(V\right.$, enter, exit, $\left.B^{\prime}, T\right)$ программы $\pi_{M}$, в которой значение функция привязки $B^{\prime}$ отличается от значения функции привязки $B$ только на вершине $v_{4}$ :

$$
B^{\prime}\left(v_{4}\right)=E, \quad B^{\prime}(v)=B(v), v \neq v_{4} .
$$

Очевидно, длины вычислений программ $\pi_{M}$ и $\pi_{M}^{\prime}$ в любой модели совпадают. Также очевидно, что результаты конечных вычислений программы $\pi_{M}^{\prime}$ в моделях вида $\mathscr{C}=(\mathscr{F}, \xi)$ всегда принадлежат множеству Err. Если известно, что результат вычисления принадлежит множеству Err, то длина вычисления однозначно определяет этот результат. 
Теорема 2. Программы $\pi_{M} u \pi_{M}^{\prime}$, построенные описанным образом для произвольной машинь Тьюринга $M$, эквивалентны на полугрупповой шкале F्F тогда и только тогда, когда вычисление этой машины Тьюринга бесконечно.

Доказательство. Если вычисление машины Тьюринга $M$ конечно, то вычисление программы $\pi_{M}$ в естественной модели конечно и имеет результат, отличный от результата вычисления программы $\pi_{M}^{\prime}$ в этой же модели.

Если вычисление машины Тьюринга бесконечно, то результат любого конечного вычисления программы $\pi_{M}$ в модели $\mathcal{M}=(\mathscr{F}, \xi)$ принадлежит множеству Err, а значит, совпадает с результатом вычисления программы $\pi_{M}^{\prime}$ в этой же модели.

Теорема 3. Существует уравновешенная полугрупповая шкала, базирующаяся на полугруппе с разрешимой проблемой равенства слов, такая, что проблема эквивалентности программ на этой шкале алгоритмически неразрешима.

\section{Список литературы}

1. Глушков В. М., Летичевский А. А., Теория дискретных преобразователей. Избранные вопросы алгебры и логики (1973) 5-39.

2. Захаров В. А., Быстрые алгоритмы разрешения эквивалентности операторных программ на уравновешенных шкалах. Математические вопросы кибернетики (1998) 7, 257-280.

3. Захаров В. А., Быстрые алгоритмы разрешения эквивалентности пропозициональных операторных программ на упорядоченных полугрупповых шкалах. Вестник Московского университета, сер. 15: Вычислительная математика и кибернетика (1999), № 3, 29-35.

4. Летичевский А. А., Эквивалентность автоматов относительно полугрупп. Теоретическая кибернетика (1970), № 6, 1-71.

5. Ляпунов А. А., О логических схемах программ. Проблемы кибернетики (1958), № 1, 46-74.

6. Подловченко Р. И., От схем Янова к теории моделей программ. Математические вопросы кибернетики (1998) 7, 281-302.

7. Подловченко Р. И., Полугрупповые модели программ. Программирование (1981), № 4, 9-19.

8. Подловченко Р. И., Система преобразований, полная в классе схем программ с перестановочными операторами. Программирование (1998), № 2, 58-67.

9. Щербина В. Л., Захаров В. А., О сложности распознавания эквивалентности машин Тьюринга без записи на ленту. В сб.: Материаль XVI Международной школь-семинара «Синтез и сложность управляющих систем». Изд-во механико-математического факультета МГУ, Москва, 2006, c. $147-150$.

10. Янов Ю. И., О логических схемах алгоритмов. Проблемы кибернетики (1958), № 1, 75-127.

Статья поступила 21.01.2009. 